\title{
Novos anticoagulantes para a profilaxia do tromboembolismo venoso em cirurgias ortopédicas de grande porte
}

\author{
New anticoagulants for the prophylaxis of venous thromboembolism
}

Ricardo de Alvarenga Yoshida', Winston Bonetti Yoshida², Hamilton de Almeida Rollo³

\begin{abstract}
Resumo
Após cerca de 50 anos de experiência com a heparina e antagonistas da vitamina $K$ (AVK), pesquisas e estudos com novos anticoagulantes vêm evoluindo de forma crescente nos últimos anos. Embora consagrados pelo uso, os anticoagulantes tradicionais têm limitações importantes em termos de controle laboratorial, complicações, efeitos colaterais, interações com medicamentos e dieta. A heparina não fracionada (HNF) tem interação com proteínas plasmáticas e parede vascular, pode desencadear trombocitopenia induzida pela heparina (TIH), só pode ser administrada por via parenteral, exige controle laboratorial pelo teste da tromboplastina parcial ativada (TTPa), pode provocar osteoporose e alopecia quando usada por períodos prolongados e sua produção tem origem biológica. A AVK tem a vantagem de poder ser ministrada por via oral, mas o controle (feito pela razão normatizada internacional) pode ser difícil em alguns casos, já que tem início de ação demorado, janela terapêutica estreita, interação com dieta e grande número de medicamentos, pode provocar necrose de pele em portadores de deficiência de antitrombina e de proteínas C e S, e pode induzir alterações fetais quando usada na gravidez. Na década de 1980, surgiram as heparinas de baixo peso molecular, que foram uma evolução da heparina não fracionada, pois apresentaram maior biodisponibilidade, dosagem por peso corporal, sem necessidade de controle laboratorial, administração por via subcutânea, menor risco de trombocitopenia induzida pela heparina, e eficácia e segurança similares à heparina não fracionada. Na última década surgiram, então, uma série de novos anticoagulantes no mercado, os quais têm apresentado resultados promissores em várias situações de profilaxia e tratamento do tromboembolismo venoso. Nesta revisão, são apresentados as novas heparinas de baixo peso molecular, as heparinas de ultrabaixo peso molecular, os pentassacarídeos, os novos inibidores diretos do fator Xa e inibidores do fator lla.
\end{abstract}

Palavras-chave: Anticoagulantes; heparina; heparina de baixo peso molecular; varfarina; revisão; tromboembolia venosa.

\begin{abstract}
After about 50 years of experience with heparin and vitamin $\mathrm{K}$ antagonists (VKA), research and clinical studies of new anticoagulants have recently evolved. Although traditional anticoagulants have proven to be clinically useful, they have important limitations in terms of laboratory control, complications, side effects and interactions with medications and food. Unfractionated heparin interacts with plasma proteins and the vascular wall, may trigger thrombocytopenia, can only be administered parenterally, requires control by the laboratory test of partial thromboplastin time, may cause osteoporosis and alopecia when used for long periods and it is produced from biological sources. VKA have the advantage of being administered orally, but the control (made by the international normalized ratio) can be difficult in some cases, since they have delayed onset of action and metabolism and a narrow therapeutic window. They also interact with foods and with a large number of medications, can cause skin necrosis in patients with antithrombin and protein C and S deficiencies and may induce fetal changes when prescribed in pregnancy. In the 1980's the low-molecular-weight heparins were developed and proved to be an evolution over unfractionated heparin, because of their greater bio-availability, fixed dose per body weight, no need for laboratory control, subcutaneous administration, lower risk of heparin-induced thrombocytopenia, and efficacy and safety similar to unfractionated heparin. Over the last decade, a series of new anticoagulants have appeared in the market and shown promising results in several situations of venous thromboembolism prophylaxis and treatment. In the present review, the new low-molecular-weight heparins, ultra-low molecular weight heparin, pentasaccharides and the new direct inhibitors of factor Xa and factor lla.are addressed.
\end{abstract}

Keywords: Anticoagulants; heparin; heparin, low-molecular-weight; warfarin; review; venous thromboembolism.

Trabalho realizado: Departamento de Cirurgia e Ortopedia da Faculdade de Medicina de Botucatu da Universidade Estadual Paulista "Júlio de Mesquita Filho" (Unesp)

'Doutor pelo Departamento de Cirurgia e Ortopedia da Faculdade de Medicina de Botucatu da Universidade Estadual Paulista "Júlio de Mesquita Filho" (Unesp); Cirurgião Vascular e Endovascular; Colaborador da Disciplina de Cirurgia Vascular e Endovascular da Faculdade de Medicina, Campus de Botucatu, da Unesp, Botucatu (SP), Brasil.

${ }_{2}^{2}$ Professor Titular da Disciplina de Cirurgia Vascular e Endovascular do Departamento de Cirurgia e Ortopedia da Faculdade de Medicina de Botucatu da Unesp; Chefe do Serviço de Cirurgia

Vascular e Endovascular do Departamento de Cirurgia e Ortopedia da Faculdade de Medicina de Botucatu da Unesp, Botucatu (SP), Brasil.

${ }_{3}^{3}$ Professor Adjunto da Disciplina de Cirurgia Vascular e Endovascular do Departamento de Cirurgia e Ortopedia da Faculdade de Medicina de Botucatu da Unesp; Chefe da Disciplina de

Cirurgia Vascular e Endovascular do Departamento de Cirurgia e Ortopedia da Faculdade de Medicina de Botucatu da Unesp, Botucatu (SP), Brasil.

Informações sobre financiamento: não houve

Conflitos de interesse: Winston Bonetti Yoshida - Consultor da Bayer do Brasil

Submetido em: 03.04.11. Aceito em: 28.05.11

J Vasc Bras. 2011;10(2):145-153 


\section{Introdução}

Após cerca de 50 anos de experiência com a heparina e antagonistas da vitamina $\mathrm{K}(\mathrm{AVK})$, pesquisas e estudos com novos anticoagulantes vêm evoluindo de forma crescente nos últimos anos. Embora consagrados pelo uso, os anticoagulantes tradicionais tem limitações importantes em termos de controle, complicações, efeitos colaterais e interações com medicamentos e dieta ${ }^{1}$.

Tanto a heparina não fracionada (HNF) como as cumarinas (AVK) estão em uso clínico, pois seus respectivos mecanismos de ação foram bem elucidados ${ }^{2}$. Os estudos pioneiros com heparina de baixo peso molecular (HBPM) foram feitos no Brasil, pela equipe do Professor Carl Peter von Dietrich ${ }^{3}$, e essa substância permanece em uso clínico desde a década de 1980, com vantagens importantes em relação à HNF, em termos de eficácia e segurança ${ }^{4}$. Entretanto, ainda tem alguma limitação pelo uso exclusivo parenteral e produção biológica.

Essas limitações deram estímulo para estudos e descobertas de novas drogas anticoagulantes, as quais foram introduzidas ao longo dos últimos 10 anos, sendo que muitas ainda estão em desenvolvimento clínico ${ }^{2}$. Na investigação por novos anticoagulantes, os pesquisadores vão buscar inspiração na natureza, em animais hematófagos, insetos e serpentes ${ }^{2}$. A identificação da estrutura molecular desses anticoagulantes acaba dando origem a pequenas moléculas que se encaixam diretamente nos sítios catalíticos das enzimas de coagulação ou agem indiretamente nesses sítios, estimulando ou interagindo com as proteínas coagulantes ${ }^{2}$.

O objetivo desta revisão é dar resposta a 3 questões fundamentais:

1. por que precisamos de novos anticoagulantes?

2. como pesquisar novas moléculas anticoagulantes?

3. quais foram os novos anticoagulantes que surgiram após o ano de 2000 ?

\section{Por que precisamos de novos anticoagulantes?}

Os anticoagulantes são considerados medicamentos indispensáveis na prevenção primária e secundária de eventos tromboembólicos arteriais e venosos e na realização de cirurgias vasculares e cardíacas ${ }^{4,5}$.

Até cerca de 20 anos atrás, a HNF e os AVK eram os únicos anticoagulantes disponíveis ${ }^{6}$. Ambos os grupos de medicamentos, HNF e AVKs, foram colocados em uso clínico mesmo antes que seus mecanismos de ação tivessem sido completamente elucidados. A heparina convencional (a HNF), descoberta em 1916 por Jay McLean, foi introduzida no tratamento do tromboembolismo venoso (TEV) em 1938. AVK vêm sendo utilizados clinicamente desde a década de $1950^{7}$.

A HNF é administrada por via parenteral (intravenosa - IV ou subcutânea - SC), exige controle laboratorial, o que a torna incômoda e dispendiosa para uso prolongado, especialmente fora do ambiente hospitalar, pois visitas de um profissional de saúde podem ser necessárias se os pacientes forem relutantes ou incapazes de se autoadministrarem ${ }^{8,9}$. Pode também causar trombocitopenia induzida pela heparina (TIH), que é uma complicação que, embora rara, é grave, fazendo-se necessária a monitorizarão das plaquetas com seu uso ${ }^{10}$. Complicações não menos importantes são a osteoporose e a alopecia ${ }^{6,11}$. Outra limitação importante das heparinas é que são produzidas por meio de matéria-prima de origem biológica, o que pode ocasionar contaminações ${ }^{12}$. Na década de 1980, o surgimento da "síndrome da vaca louca", na Inglaterra, fez com que a produção de HNF fosse transferida de bovinos para intestinos de porcos. Além disso, recentemente, houve um problema sério de contaminação das HNF por sulfato de dermatana superfosfatada, que ocasionou mortes no mundo todo ${ }^{12}$. Apesar dessas limitações, as indicações das heparinas estão bem estabelecidas em prevenção primária e tratamento do TEV, síndromes coronarianas agudas, anticoagulação plena em cirurgia vascular e endovascular, circulação extracorpórea e hemodiálise ${ }^{11}$.

A AVK tem a vantagem de poder ser ministrada por via oral, mas, por outro lado o controle (feito pela razão normatizada internacional) pode ser difícil em alguns casos já que possui janela terapêutica estreita, pode haver interação com dieta e grande número de medicamentos, necessitando acompanhamento rigoroso para ajuste da dose no sentido de se assegurar que os efeitos anticoagulantes permaneçam no intervalo terapêutico ${ }^{5,6,8,13-16}$. Além disso, pode provocar necrose de pele em portadores de deficiência de antitrombina e de proteínas $\mathrm{C}$ e $\mathrm{S}$ e pode induzir alterações fetais quando usada na gravidez ${ }^{5,6,8,13-16}$. Mesmo assim, esses medicamentos são muito eficazes na prática clínica, do ponto de vista de seu uso prolongado ${ }^{9,17}$, e suas indicações estão bem estabelecidas em: prevenção de acidente vascular cerebral (AVC) em pacientes com fibrilação atrial (FA) e em pacientes com válvulas cardíacas, tratamento do TEV em pacientes com trombose venosa profunda (TVP), prevenção primária do TEV em pacientes submetidos à cirurgia ortopédica nos EUA ${ }^{18,19}$.

Na década de 1980, apareceram as HBPM, a partir da fragmentação ou despolimerização da heparina por via química ou processo enzimático. Vale salientar que as primeiras pesquisas com o fracionamento da heparina foram feitas por uma equipe de brasileiros, liderada pelo Professor Carl Peter von Dietrich da Univerisade Federal de São Paulo (Unifesp) ${ }^{3}$. 
Com o processamento da HNF (PM entre 4 e 40.000 Dalton), origina-se a HBPM com PM médio de 5.000 Dalton. Com essa configuração, a HBPM passa a ter ação predominantemente anti-Xa (relação média: 2,5/1), maior biodisponibilidade, dispensa de monitoramento laboratorial, o que possibilita o tratamento domiciliar ${ }^{20}$. Tem indicações bem estabelecidas no profilaxia e tratamento do $\mathrm{TEV}$, na síndrome coronariana aguda, no AVC trombótico, nos transplantes e, inclusive, em pacientes que se encontram em tratamento de câncer ${ }^{21}$. Apesar de consagrada, tem limitações por ter produção de origem biológica e por ser injetável somente.

Posteriormente, surgiram outros anticoagulantes como a hirudina (isolada a partir da saliva das sanguessugas Hirudo medicinalis), formas recombinantes da hirudina (a lepirudina -Refludan, a desirudina - Iprivask, Revasc], derivados da hirudina (bivaluridina - Angiomax), o danaparoide (Orgaran - mistura de glicosaminoglicanos com $84 \%$ de sulfato de heparana, $12 \%$ de sulfato de dermatana e $4 \%$ de sulfato de condroitina) que foram aprovados em algumas situações clínicas, mas o custo e o risco hemorrágico constituíram barreiras para disseminação rotineira na prática clínica ${ }^{4}$. Portanto, atualmente, as opções de tratamento anticoagulantes clássicos em nosso meio são a HNF, HBPM, AVKs e, mais recentemente, o pentassacarídeo sintético fondaparinux (Arixtra). Embora todas essas drogas tenham se mostrado eficazes no tratamento e na redução do risco da doença tromboembólica ao longo do tempo, elas estariam, por outro lado, associadas a inconvenientes, que limitam seu uso e sua ampla aceitação clínica9 ${ }^{9}$. Dessa maneira, apesar da eficácia desses anticoagulantes, seu risco hemorrágico, a restrição de vias de administração, a necessidade de controle laboratorial rigoroso e frequente têm levado à busca de um anticoagulante ideal, que, além de ser eficaz, deveria ter menor risco hemorrágico, ausência de efeitos colaterais, de interação com outras medicações ou alimentos, ser de fácil administração, confortável para o paciente e para equipe médica, sem necessidade de controle laboratorial, de baixo custo e com antídoto ${ }^{1}$.

\section{Como pesquisar novos anticoagulantes?}

De modo geral, a fonte de inspiração para criação de novas moléculas encontra-se na natureza ${ }^{2}$. A própria HNF foi descoberta a partir de pesquisas em fígado de cães, de onde foi extraída pela primeira vez. Depois, observou-se que outras fontes para sua produção poderiam ser os pulmões e intestinos de mamíferos. Atualmente, a HNF é produzida principalmente a partir de intestinos de porcos, sendo a China um dos maiores produtores mundiais de matéria prima ${ }^{12}$.
As AVKs, como outro exemplo de fontes naturais, foram descobertas a partir da morte por hemorragia do gado que se alimentava da planta trevo doce (Melilotus officinalis). Na década de 1920, Frank Schofield, um patologista veterinário descobriu que somente o gado que se alimentava com silagem com trevo doce apresentava a hemorragia. Em 1933, o químico Karl Paul Gerhardt Link isolou o anticoagulante presente no trevo doce, o qual denominou de dicumarol ${ }^{22}$. Após várias pesquisas chegou ao warfarin (acrônimo de Wisconsin Alumni Research Foundation), o qual foi patenteado para uso como raticida, sendo posteriormente aprovado para uso como medicação anticoagulante em humanos em $1954^{1}$.

A hirudina foi concebida a partir de estudos da saliva das sanguessugas (Hirudo medicinalis) e serviu de fonte de inspiração para um série de moléculas com base em sua ação inibidora nos sítios de ligação da trombina. Da mesma forma, a estrutura do anticoagulante pentassacarídeo foi baseada na sequência de 5 sacárides responsáveis pela ação da heparina ativando a antitrombina ${ }^{4}$.

Com relação a interação proteína-proteína, uma vez conhecida a sequência de aminoácidos que compõe a região do inibidor e/ou substrato da enzima, podem ser obtidas moléculas sintéticas ou recombinantes que simulem essa interação, inibindo, dessa forma, essa ação enzimática. Pelo estudo de composição e geometria do sítio ativo das enzimas, é possível bloquear a atividade enzimática por agentes químicos. As moléculas com sítio ativo bloqueadas continuam estruturalmente similares às enzimas de origem, competindo pelo mesmo sítio de ligação das mesmas, não havendo, assim, continuidade das reações que levam à formação do trombo. Outra forma de inibição é quando oligo ou polissacarídeos se ligam a inibidores naturais presentes na coagulação sanguínea, causando mudança conformacional, de forma a aumentar a afinidade e a velocidade de interação enzima-inibidor.

Outras fontes importantes para pesquisa são: algas, carrapatos, nematoides, lagartas, além dos sanguessugas e vegetais (Figura 1).

A partir do isolamento da molécula "mãe", a indústria farmacêutica tem equipamentos computadorizados para criar uma infinidade de variações na estrutura molecular, da ordem de 200.000 compostos, as quais ficam armazenadas em uma "biblioteca de moléculas" potenciais. Equipamentos de robótica fazem testes in vitro de cada uma delas em tempo recorde, apontando as moléculas mais promissoras. Estas são em seguida testadas in vivo, em modelos animais de trombose venosa ou arterial ${ }^{23-26}$, privilegiando aquelas com ação mais proeminente e com biodisponibilidade, farmacodinâmica e farmacocinética mais apropriadas. Novos testes em animais são necessários para certificar a segurança em termos de toxicidade ${ }^{27}$. Para 


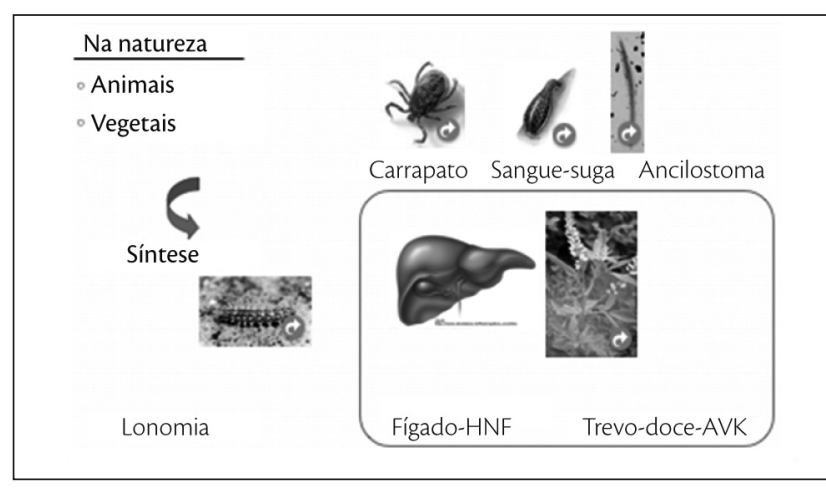

Figura 1 - Fontes encontradas na natureza para pesquisa de novos anticoagulantes.

tanto, são estudados em vários modelos animais (diversas espécies como camundongo, rato, cobaia e coelho). De 1.000 substâncias testadas em animais, somente 10 são aprovadas para continuar em desenvolvimento seguindo para Fase I da pesquisa clínica ${ }^{28}$.

Depois disso, as drogas selecionadas são testadas em pessoas normais, por meio de estudos denominados de "Fase I", visando conhecer absorção, efeitos colaterais, biodisponibilidade, farmacodinâmica e farmacocinética. Posteriormente, estudos chamados de "Fase II" são usados para testes de doses da nova molécula, testados em um pequeno grupo de pacientes com problemas tromboembólicos, visando estudar escalonamento de doses, eficácia e segurança. O grande teste virá com estudos "Fase III", nos quais um grande contingente de pacientes é tratado em testes de eficácia e segurança, em comparação ao tratamento padrão. Os estudos "Fase IV" são feitos para testes de tolerância, eficácia e segurança, com a droga já no mercado (farmacovigilância). No lançamento, o perfil do novo medicamento já foi avaliado em condições ideais, em pacientes sob estrito acompanhamento médico e geralmente sem doenças associadas. Porém, por muitos anos, ainda são feitas pesquisas em hospitais e laboratórios de todo o mundo para se saber se pessoas com outras doenças ou condições especiais podem também usá-lo, pois devem ainda ser observadas as interações inesperadas, as reações adversas e os limites de uso seguro do medicamento.

O desenvolvimento de um medicamento é um processo longo, trabalhoso e de custo bastante elevado. Em geral, de cada 10.000 moléculas identificadas com potencial terapêutico, somente 1.000 chegam à fase de investigação pré-clínica. Dessas, apenas 10 são estudadas em seres humanos e só uma delas chega ao mercado, após aprovação e registro para uso terapêutico. Esse processo tem duração de aproximadamente 10 anos e custo em torno de 2 bilhões de reais ${ }^{29}$. Estudos clínicos devem ser conduzidos de forma profissional, ética e cientificamente corretos ${ }^{30}$.

Em resumo, para busca de novos anticoagulantes, o conhecimento molecular e estrutural, o mecanismo de ação dos fatores de coagulação e suas interações com componentes sanguíneos são essenciais para elaboração e desenvolvimentos de novas drogas.

\section{Quais são os novos anticoagulantes?}

Nesta seção, são abordados os novos anticoagulantes mais promissores que despontaram após o ano 2000, privilegiando aqueles que completaram testes de Fase III de avaliação de profilaxia em cirurgia ortopédica (Tabela 1).

Tabela 1 - Novos anticoagulantes, laboratório produtor, nível de ação na cascata da coagulação, via de administração, meia-vida (em horas), vias de excreção, fases dos estudos e disponibilidade de antídotos

\begin{tabular}{|c|c|c|c|c|c|c|c|c|}
\hline Anticoagulante & Laboratório & $\begin{array}{l}\text { Fase da } \\
\text { cascata }\end{array}$ & $\begin{array}{c}\text { Via de } \\
\text { administração }\end{array}$ & Ação & $\begin{array}{c}\text { Meia-vida } \\
\text { (horas) }\end{array}$ & Excreção & Estudos & Antídoto \\
\hline Bemiparina & ROVI & $\mathrm{Xa}$ & SC & Indireta & 5 & Renal & Fase III & Não \\
\hline Semuloparina & SANOFI-AVENTIS & $\mathrm{Xa}$ & SC & Indireta & 11 & Renal & Fase III & Não \\
\hline Fondaparinux & Glaxo-Smith-Kline & $\mathrm{Xa}$ & SC & Indireta & 17 & Renal & Fase IV & Não \\
\hline Idrabiotaparinux & SANOFI-AVENTIS & $\mathrm{Xa}$ & SC & Indireta & $80-130$ & Renal & Fase III & Avidina \\
\hline Rivaroxabana & BAYER SHERING & $\mathrm{Xa}$ & VO & Direta & $4-9$ & Renal / Hepática & Fase IV & Não \\
\hline Apixabana & Bristol-Myers Squibb & $\mathrm{Xa}$ & VO & Direta & $8-15$ & Renal / Intestinal & Fase III & Não \\
\hline Edoxabana & DAIISHI SANKIO & $\mathrm{Xa}$ & VO & Direta & $6-11$ & Renal & Fase III & Não \\
\hline Betrixaban & PORTOLA & $\mathrm{Xa}$ & VO & Direta & 19 & Hepática & Fase II & Não \\
\hline Dabigatrana & BOERINGER INGELHEIM & Ila & VO & Direta & $14-17$ & Renal / Hepática & Fase IV & Não \\
\hline YM-150 & ASTELLAS & $\mathrm{Xa}$ & VO & Direta & $14-20$ & Renal & Fase II & - \\
\hline TAK-442 & TAKEDA & $\mathrm{Xa}$ & VO & Direta & $9-13$ & Renal & Fase II & - \\
\hline Otamixabana & SANOFI-AVENTIS & $\mathrm{Xa}$ & EV & Direta & 0,5 & Renal / Hepática & Fase III & - \\
\hline aZD-0837 & ASTRA-ZENECA & Ila & VO & Direta & - & - & Fase II & - \\
\hline MCC-977 & MITSUBISHI & Ila & VO & Direta & - & - & Fase II & - \\
\hline
\end{tabular}

SC: subcutânea; VI: via oral; EV: endovenosa. 
Há um debate na literatura sobre qual seria o melhor sítio de ação na cascata de coagulação (Figura 2) para desenvolvimento de novos anticoagulantes: inibidores da fase inicial, da fase de propagação ou da fase final da

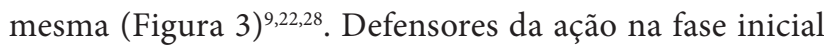
da cascata de coagulação argumentam que as doses necessárias para inibição do processo de coagulação nesse sítio seriam menores em relação às demais fases da cascata, o que poderia, em contrapartida, implicar menor risco de sangramentos. Por outro lado, muitos pesquisadores defendem que a inibição do fator X (fase de propagação) seria um alvo estratégico para ação anticoagulante, uma vez que este fator é chave na cascata de coagulação, por ser a intersecção das fases intrínseca e extrínseca da mesma e as doses necessárias para exercer sua ação também seriam menores. Além disso, haveria menor interferência em ações adicionais da trombina sobre o processo inflamatório, sistema complemento e regulação do processo de coagulação por meio de ações regulatórias adicionais sobre os fatores V, VIII, XI, XIII, trombomodulina, proteína $\mathrm{C}$ e agregação plaquetária. Segundo esses autores, a inibição direta da trombina poderia prejudicar estes mecanismos9. O fato é que ainda não se tem um consenso sobre essas ponderações e que vários anticoagulantes, cuja ação é antitrombínica direta, têm sido testados com sucesso em termos de eficiência e segurança ${ }^{31}$.

\section{Inibidores indiretos do fator Xa}

Recentemente tem sido pesquisadas novas HBPM, com PM médio cada vez menores, chamadas de segunda geração, o que acarreta alterações nas características farmacocinéticas e farmacodinâmicas. Para serem assim chamadas, tem que apresentar $\mathrm{PM}<4.000$ Dalton e menos de $15 \%$ de cadeias maiores que 6.000 Dalton, com relação Anti Xa/IIa $>4: 1^{32}$. A bemiparina se encaixa nesse perfil, e tem $96 \%$ de biodisponibilidade, meia-vida de 5,3 horas e foi testada em Fase III na profilaxia do TEV em cirurgia ortopédica maior ${ }^{33}$. Em estudo de não inferioridade em relação à enoxaparina, com 333 pacientes submetidos à artroplastia total de joelho ${ }^{34}$, o grupo bemiparina teve $32,1 \%$ de TEV e o grupo enoxaparina teve $36,9 \%$. O sangramento no sítio operatório foram similares (22,7 versus $32,5 \%$, respectivamente). A amostra pequena não permitiu análises mais detalhadas de outros parâmetros.

Ainda dentro da classe das heparinas, foram criadas as heparinas de ultrabaixo peso molecular (HUBPM). Para pertencer a esse grupo, estas tem que ter PM entre 2.000 a 3.000 Dalton e relação Xa/IIa de 30:135,36. Estão representadas, principalmente, pela semuloparina da

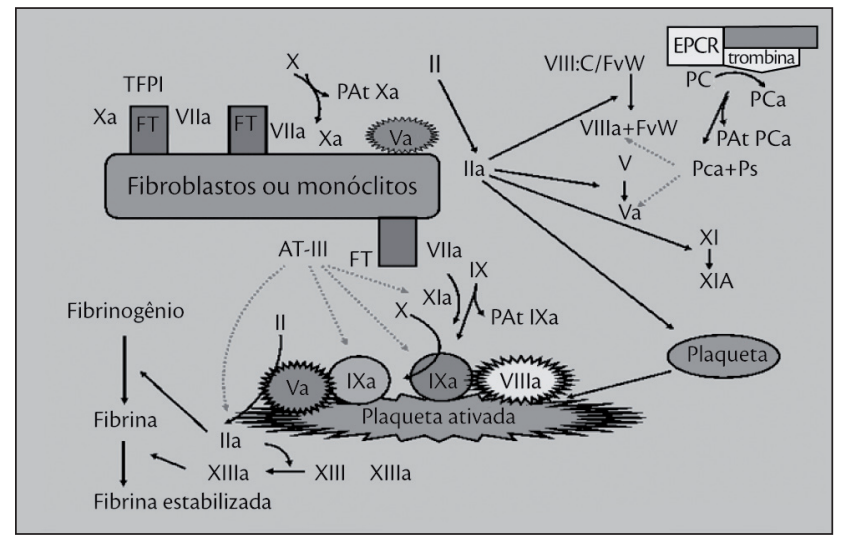

Figura 2 - Cascata de coagulação resumida.

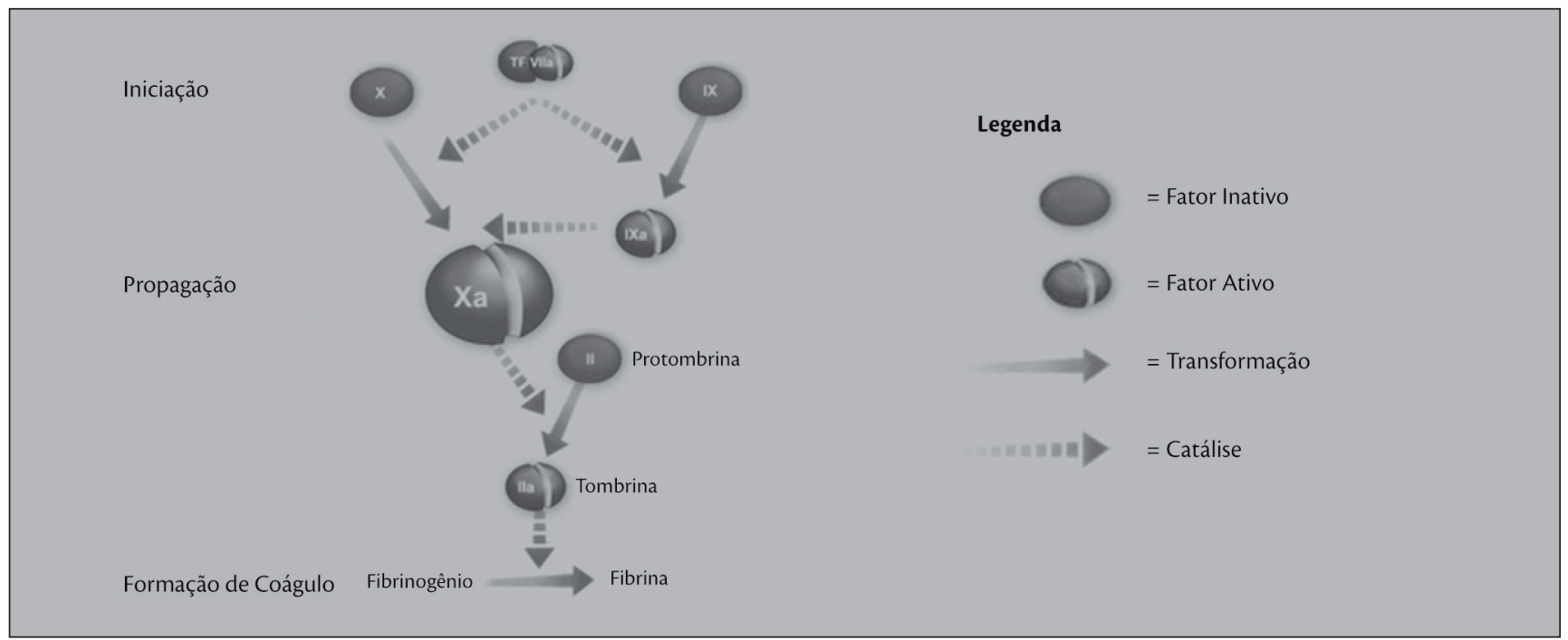

Figura 3 - Fases da cascata de coagulação resumidas. 
Sanofi-Aventis e RO14 do Laboratório Rovi". A semuloparina $(20 \mathrm{mg} / \mathrm{d})$, particularmente, foi testada em três situações de profilaxia de cirurgia ortopédica maior, incluindo fratura de quadril, prótese total de quadril e de joelho ${ }^{37}$, em comparação com enoxaparina nas doses de $30 \mathrm{mg}$ duas vezes por dia ou $40 \mathrm{mg}$ uma vez por dia. A meta-análise destes três estudos mostrou eficácia primária superior (qualquer TEV e mortalidade) e segurança similar $^{37}$, em relação à enoxaparina. As demais drogas dessa classe ainda estão em Fase III de testes clínicos

Os pentassacarídeos constituem a menor molécula (sequência de cinco sacárides) derivada da heparina e que podem ativar a antitrombina. Têm a vantagem de serem sintéticos, mas com limitações relacionadas à administração, exclusivamente via parenteral. $\mathrm{O}$ fondaparinux (Arixtra da Glaxo-Smith-Kline), idraparinux (SanofiAventis) e idrabiotaparinux (Sanofi-Aventis) são os representantes desse grupo, dentre os quais o fondaparinux tem sido amplamente recomendado para profilaxia de $\mathrm{TEV}^{21,38}$. $\mathrm{O}$ fondaparinux liga-se seletivamente à antitrombina, catalisando a inibição do fator $\mathrm{Xa}$, não se liga às plaquetas (não provoca TIH) e tem meia-vida de 17 horas $^{38}$. Em meta-análise de quatro estudos randomizados, o fondaparinux mostrou redução geral de $50 \%$ de eventos primários (TEV) até o $11^{\circ}$ dia, mas às custas de maior frequência de sangramento ${ }^{39}$. O idraparinux e idrobiotaparinux são formas hipermetiladas do fondaparinux, têm meias-vidas mais prolongadas ( 80 a 130 horas), de modo que podem ser usados em intervalos de 1 semana, ministradas por via $\mathrm{SC}^{28}$. O idrabiotaparinux tem biotina incorporada à molécula, de modo que sua ação anticoagulante pode ser revertida com injeção de avidina ${ }^{40}$. Entretanto, devido ao maior risco de sangramento observado em estudo de tratamento do $\mathrm{TEV}^{41}$ e à inferioridade em relação à enoxaparina + varfarina no tratamento da embolia pulmonar $(\mathrm{EP})^{42}$, aparentemente ambas não são mais desenvolvidas pelo fabricante.

\section{Inibidores diretos do fator $\mathrm{Xa}$}

Dentro desse grupo, está a rivaroxabana, derivada da oxazilidinona, produzida pelo Laboratório Bayer Healthcare, a apixabana do laboratório Bristol Meyer Squibb e a edoxabana, produzida pelo Laboratório Daiichi-Sankyo ${ }^{28}$. São moléculas pequenas, com cerca de 500 Dalton e com potente ação direta anti-Xa, porém a maioria deles não tem antídoto.

A rivaroxabana atinge níveis plasmáticos em aproximadamente 3 horas, com meia-vida de 4 a 9 horas $^{43}$. A biodisponibilidade é de $80 \%$, tem pouca interação medicamentosa, não tem interferência com a dieta em sua absorção, e 66\% são eliminados pelos rins ${ }^{28}$. Foi testada em profilaxia do TEV em cirurgia ortopédica maior em quatro grandes estudos denominados de RECORD, em comparação com a enoxaparina nas doses de $40 \mathrm{mg}$ uma vez por dia (esquema europeu) ${ }^{44-46}$ ou $30 \mathrm{mg}$ duas vezes por dia (esquema norte-americano ${ }^{47}$. Análise conjunta dos 4 estudos $^{48}$, incluindo 12.500 pacientes, mostrou que houve redução de cerca de $50 \%$ no risco de TEV ou morte, sem diferença estatística com relação ao sangramento. O medicamento foi aprovado para uso em profilaxia do TEV após cirurgia ortopédica maior na Europa, Canadá, América Latina e Brasil ${ }^{49}$.

A apixabana (Bristol Meyer Squibb) é rapidamente absorvida por via oral, com pico de ação em 3 horas. Sua meia-vida é de 8 a 11 horas quando ministrada em duas vezes por dia e de 12 a 15 horas em dose única. Sua absorção não tem interferência da dieta, tem biodisponibilidade de $50 \%$ e eliminação renal (25 a $30 \%)$ e fecal $(65 \%)^{28}$. Estudos de profilaxia em cirurgia ortopédica maior mostraram não inferioridade quanto à eficácia primária em relação ao esquema europeu de dosagem de enoxaparina (40 mg uma vez por dia $)^{50}$, mas não atingiram não inferioridade em relação ao esquema norte-americano de enoxaparina (30 mg duas vezes por dia $)^{51,52}$. Os resultados de segurança (sangramentos) foram similares ${ }^{28}$.

A edoxabana (Daiichi-Sankyo) terminou estudo Fase III de profilaxia em cirurgia ortopédica maior (prótese total de joelho), comparando com enoxaparina $20 \mathrm{mg}$ a cada 12 horas $^{53}$. Os resultados de eficácia foram superiores à enoxaparina neste esquema de dosagem e os de segurança (sangramentos) foram similares. Esse estudo ainda não publicado.

Ainda em fase de estudos está o betrixabana (Laboratório Portola), com diferencial de ter antídoto para o fator Xa. O estudo EXPERT finalizou Fase II em pacientes submetidos à cirurgia do joelho, em comparação com enoxaparina na dose de $30 \mathrm{mg}$ duas vezes por dia (esquema norte americano). Os resultados com duas doses diferentes de betrixabana foram promissores ${ }^{54}$.

Além destes, estão em avaliação YM-150 (Astellas), TAK-442 (Takeda) e otamixabana (Sanofi-Aventis) ${ }^{43}$.

\section{Inibidores do fator Ila}

Nesse grupo, enquadra-se a dabigatrana (Boehringer Ingelheim). Esta inibe tanto a trombina livre quanto a ligada ao trombo. O etexilato de dabigatrana é uma pró-droga, 
que se converte em dabigatrana logo após administração oral, após passagem pelo fígado, com pico de concentração em 1,5 horas e meia-vida é de 14 a 17 horas. A biodisponibilidade é de 7,5\% e a eliminação da dabigatrana não absorvida é principalmente pelas fezes. Já dabigatrana circulante (ativada no fígado) é eliminada pelos rins $(80 \%)$. Pode ocorrer interação medicamentosa com quinina/quinidina e verapamil e não foi usada em pacientes com insuficiência renal em seus estudos estudos ${ }^{43}$.

A dabigatrana foi testada em profilaxia de cirurgia ortopédica maior nos estudos denominados RE-Novate, RE-Model e RE-Mobilize ${ }^{55}$. Os dois primeiros envolveram cirurgias do quadril e joelho, respectivamente, comparando essa droga com enoxaparina na dose de 40 mg uma vez por dia (esquema europeu). O estudo REMobilize foi feito em pacientes submetidos à cirurgia do joelho, comparando a dabigatrana com enoxaparina na dose de $30 \mathrm{mg}$ duas vezes por dia (esquema americano). Em todos os estudos foram usadas duas doses de dabigatrana (150 e $220 \mathrm{mg}$ ao dia). O estudo RE-Novate II comparou as duas doses de dabigatrana com enoxaparina $40 \mathrm{mg}$ uma vez por dia em pacientes submetidos à cirurgia do quadril. Meta-análise dos estudos RE-Model e RE-Novate mostrou que a dabigatrana foi não inferior à enoxaparina $40 \mathrm{mg}$ uma vez por dia (esquema europeu), sendo o perfil de segurança similar. Com relação ao estudo RE-Mobilize, no qual a comparação foi feita com dose de $30 \mathrm{mg}$ duas vezes por dia de enoxaparina (esquema europeu), a eficácia primária da dabigatrana foi inferior ao grupo da enoxaparina ${ }^{55}$.

Estão ainda em fases iniciais de estudos os inibidores do fator IIa: aZD-0837 (Astra- Zeneca) e MCC-977 (Mitsubishi) $^{43}$.

\section{Conclusão}

Os estudos até agora realizados mostram que os novos anticoagulantes orais podem constituir alternativas para as AVKs em pacientes de risco para TEV. Entretanto, ainda se está longe de um anticoagulante ideal, cujas características seriam: eficiência primária superior, ausência de interação com drogas ou dieta, possibilidade de diferentes vias de administração, grande biodisponibilidade, não atravessar barreira placentária, sem necessidade de controle laboratorial, não provocar trombocitopenia, ser de origem sintética, de fácil obtenção, baixo custo, não alterar enzimas hepáticas e com antídoto. Além disso, ser eficaz e seguro tanto na profilaxia quanto no tratamento.

\section{Referências}

1. Haas S. New anticoagulants - towards the development of an "ideal" anticoagulant. Vasa. 2009;38:13-29.

2. Hirsh J, O'Donnell M, Eikelboom JW. Beyond unfractionated heparin and warfarin: current and future advances. Circulation. 2007;116:552-60.

3. Bianchini P, Osima B, Parma B, Nader HB, Dietrich CP, Casu B et al. Fractionation and structural features of two heparin families with high antithrombotic, antilipemic and anticoagulant activities. Arzneimittelforschung. 1985;35:1215-9.

4. Oliveira L, Franco R. Novas drogas anticoagulantes. Medicina, Ribeirão Preto. 2001;34:276-81.

5. Laux V, Perzborn E, Heitmeier S, von Degenfeld G, DittrichWengenroth E, Buchmuller A, et al. Direct inhibitors of coagulation proteins - the end of the heparin and low-molecular-weight heparin era for anticoagulant therapy? Thromb Haemost. 2009;102:892-9.

6. Benmira S, Banda ZK, Bhattacharya V. Old versus new anticoagulants: focus on pharmacology. Recent Pat Cardiovasc Drug Discov. 2010;5:120-37.

7. Garcia D. Novel anticoagulants and the future of anticoagulation. Thromb Res. 2009;123 Suppl 4:S50-5.

8. Weitz J. New oral anticoagulants in development. Thromb Haemost. 2010;103:62-70.

9. Turpie AG. Oral, direct factor Xa inhibitors in development for the prevention and treatment of thromboembolic diseases. Arterioscler Thromb Vasc Biol. 2007;27:1238-47.

10. Warkentin TE, Greinacher A, Koster A, Lincoff AM. Treatment and prevention of heparin-induced thrombocytopenia: American College of Chest Physicians Evidence-Based Clinical Practice Guidelines (8th Edition). Chest. 2008;133:340S-380S.

11. Hirsh J, Bauer KA, Donati MB, Gould M, Samama MM, Weitz Jl. Parenteral anticoagulants: American College of Chest Physicians Evidence-Based Clinical Practice Guidelines (8th Edition). Chest. 2008;133:141S-159S

12. Cavalheiro-Filho C, Chamone Dde A, Rached RA, Maffei FH. Heparins - current status. Rev Assoc Med Bras. 2008;54:471-2.

13. Bauer KA. New oral anticoagulants in development: potential for improved safety profiles. Rev Neurol Dis. 2010;7:1-8.

14. Haas S. New oral Xa and Ila inhibitors: updates on clinical trial results. J Thromb Thrombolysis. 2008;25:52-60.

15. Spyropoulos AC. Brave new world: the current and future use of novel anticoagulants. Thromb Res. 2008;123 Suppl 1:S29-35.

16. Borlina LP, Cavalcantie Silva EL, Ghislandi C, TimiJRR. Conhecimento sobre anticoagulantes orais e seu manejo por médicos de pronto atendimento. J Vasc Bras. 2010;9(2):24-28.

17. Eikelboom JW, Weitz Jl. New anticoagulants. Circulation. 2010;121:1523-32.

18. Kearon C, Kahn SR, Agnelli G, Goldhaber S, Raskob GE, Comerota AJ. Antithrombotic therapy for venous thromboembolic disease: American College of Chest Physicians Evidence-Based Clinical Practice Guidelines (8th Edition). Chest. 2008;133:454S-545S. 
19. Ansell J, Hirsh J, Hylek E, Jacobson A, Crowther M, Palareti G. Pharmacology and management of the vitamin $K$ antagonists: American College of Chest Physicians Evidence-Based Clinical Practice Guidelines (8th Edition). Chest. 2008;133:160S-198S.

20. Buller HR, Agnelli G, Hull RD, Hyers TM, Prins MH, Raskob GE. Antithrombotic therapy for venous thromboembolic disease: the Seventh ACCP Conference on Antithrombotic and Thrombolytic Therapy. Chest. 2004;126:401S-428S.

21. Hirsh J, Guyatt G, Albers GW, Harrington R, Schunemann HJ. Executive summary: American College of Chest Physicians Evidence-Based Clinical Practice Guidelines (8th Edition). Chest. 2008;133:71S-109S.

22. Apostolakis S, Lip GY, Lane DA, Shantsila E. The Quest for New Anticoagulants: From Clinical Development to Clinical Practice. Cardiovasc Ther. 2010:1-11.

23. Mattar L, Maffei FH, Lastoria S, Yoshida WB, Rollo HA, Fabris VE, et al. Comparative study of the action of topical heparinoids on the evolution of experimental thrombophlebitis. Braz J Med Biol Res. 1988;21:1047-8

24. Maffei FH, Rollo HA, Fabris VE. Prevention of experimental venous thrombosis induced by contrast medium in the rat. Acta Radiol Diagn (Stockh). 1980;21:249-52.

25. Rollo HA, Maffei FH, Yoshida WB, Lastoria S, Curi PR, Mattar L. Heparin, heparin plus ASA and dipyridamole, and arteriovenous fistula as adjuvant methods to prevent rethrombosis after venous thrombectomy. Experimental study in rabbits. Int Angiol. 1991;10:88-94.

26. Maffei FH, Pinto AM, Fabris VE, Lastoria S, Rollo HD. [Experimental venous thrombosis in guinea pigs: effect of heparin and drugs affecting platelet function (author's transl)]. Rev Bras Pesqui Med Biol. 1977;10:369-78.

27. Harenberg J, Kakkar A, Bergqvist D, Barrowcliffe T, Casu B, Fareed J, et al. Recommendations on biosimilar low-molecular-weight heparins. J Thromb Haemost. 2009;7:1222-5.

28. Samama MM, Gerotziafas GT. Newer anticoagulants in 2009. J Thromb Thrombolysis. 2010;29:92-104.

29. Daiichi-Sankyo Farmacêutica [Internet]. Etapas de desenvolvimento de um novo fármaco. 20101 [citado 2011 Jun. 7]. Disponível em: http://www.daiichisankyo.com.br/PandD/EtapasDesenv.aspx

30. El Dib RP. Como praticar a medicina baseada em evidências. I Vasc Bras. 2007;6:1-4.

31. Weitz J. Factor Xa or thrombin: is thrombin a better target? J Thromb Haemost. 2007;5 Suppl 1:65-7.

32. Kakkar VV. Treatment of venous thromboembolism: experience with bemiparin. Pathophysiol Haemost Thromb. 2002;32: 406-7.

33. Martinez-Gonzalez J, Vila L, Rodriguez C. Bemiparin: second-generation, low-molecular-weight heparin for treatment and prophylaxis of venous thromboembolism. Expert Rev Cardiovasc Ther. 2008;6:793-802.

34. Navarro-Quilis A, Castellet E, Rocha E, Paz-Jimenez J, Planes A. Efficacy and safety of bemiparin compared with enoxaparin in the prevention of venous thromboembolism after total knee arthroplasty: a randomized, double-blind clinical trial. J Thromb Haemost. 2003;1:425-32.
35. Gomez-Outes A, Lecumberri R, Pozo C, Rocha E. New anticoagulants: focus on venous thromboembolism. Curr Vasc Pharmacol. 2009;7:309-29.

36. Kakkar AK. Low- and ultra-low-molecular-weight heparins. Best Pract Res Clin Haematol. 2004;17:77-87.

37. Turpie AGG, Agnelli G, Fisher W, George D, Kakkar AV, Lassen MR, et al. Benefit-to-risc profile of the ultra-molecular weight heparin (ULMWH) semuloparin for prevention of venous thromboembolism (VTE): a meta-analysis. Pathophysiol Haemost Thromb. 2010;37:OC332.

38. Reynolds NA, Perry CM, Scott LJ. Fondaparinux sodium: a review of its use in the prevention of venous thromboembolism following major orthopaedic surgery. Drugs. 2004;64:1575-96.

39. Turpie AG, Bauer KA, Eriksson BI, Lassen MR. Fondaparinux vs enoxaparin for the prevention of venous thromboembolism in major orthopedic surgery: a meta-analysis of 4 randomized double-blind studies. Arch Intern Med. 2002;162:1833-40.

40. Paty I, Trellu M, Destors JM, Cortez P, Boelle E, Sanderink G. Reversibility of the anti-FXa activity of idrabiotaparinux (biotinylated idraparinux) by intravenous avidin infusion. J Thromb Haemost. 2010;8:722-9.

41. Harenberg J, Vukojevic Y, Mikus G, Joerg I, Weiss C. Long elimination half-life of idraparinux may explain major bleeding and recurrent events of patients from the van Gogh trials. I Thromb Haemost. 2008;6:890-2.

42. Buller HR, Cohen AT, Davidson B, Decousus H, Gallus AS, Gent M, et al. Idraparinux versus standard therapy for venous thromboembolic disease. N Engl J Med. 2007;357:1094-104.

43. Garcia D, Libby E, Crowther MA. The new oral anticoagulants. Blood. 2010;115:15-20.

44. Lassen MR, Bauer KA, Eriksson BI, Turpie AG. Postoperative fondaparinux versus preoperative enoxaparin for prevention of venous thromboembolism in elective hip-replacement surgery: a randomised double-blind comparison. Lancet. 2002;359:1715-20.

45. Lassen MR, Ageno W, Borris LC, Lieberman JR, Rosencher N, Bandel TJ, et al. Rivaroxaban versus enoxaparin for thromboprophylaxis after total knee arthroplasty. N Engl J Med. 2008;358:2776-86.

46. Eriksson BI, Borris LC, Friedman RJ, Haas S, Huisman MV, Kakkar AK, et al. Rivaroxaban versus enoxaparin for thromboprophylaxis after hip arthroplasty. N Engl J Med. 2008;358:2765-75.

47. Turpie AG, Lassen MR, Davidson BL, Bauer KA, Gent M, Kwong $L M$, et al. Rivaroxaban versus enoxaparin for thromboprophylaxis after total knee arthroplasty (RECORD4): a randomised trial. Lancet. 2009;373:1673-80.

48. Turpie AG, Lassen MR, Eriksson Bl, Gent M, Berkowitz SD, Misselwitz $F$, et al. Pooled analysis of four rivaroxaban studies: effects on symptomatic events and bleeding. I Thromb Haemost. 2009;7:Abs OC-WE-004.

49. Zikria JC, Ansell J. Oral anticoagulation with factor Xa and thrombin inhibitors: on the threshold of change. Curr Opin Hematol. 2009; 16:347-56.

50. Lassen MR, Raskob GE, Gallus A, Pineo G, Chen D, Hornick P. Apixaban versus enoxaparin for thromboprophylaxis after knee replacement (ADVANCE-2): a randomised double-blind trial. Lancet. 2010;375:807-15. 
51. Lassen MR, Raskob GE, Gallus A, Pineo G, Chen D, Portman RJ. Apixaban or enoxaparin for thromboprophylaxis after knee replacement. N Engl J Med. 2009;361:594-604.

52. Lassen MR, Davidson BL, Gallus A, Pineo G, Ansell J, Deitchman D. The efficacy and safety of apixaban, an oral, direct factor Xa inhibitor, as thromboprophylaxis in patients following total knee replacement. J Thromb Haemost. 2007;5:2368-75.

53. Fuji T, Wang C), Fujita S, Tachibana S, Kawai Y, Koretsune Y, et al. Edoxaban versus enoxaparin for thromboprophylaxis after knee arthroplasty: the Stars E-3 trial. Pathophysiol Haemost Thromb. 2010;37:OC297.

54. Turpie AG, Bauer KA, Davidson BL, Fisher WD, Gent M, Huo MH, et al. A randomized evaluation of betrixaban, an oral factor Xa inhibitor, for prevention of thromboembolic events after total knee replacement (EXPERT). Thromb Haemost. 2009;101:68-76.

55. Wolowacz SE, Roskell NS, Plumb JM, Caprini JA, Eriksson BI Efficacy and safety of dabigatran etexilate for the prevention of venous thromboembolism following total hip or knee arthroplasty. A meta-analysis. Thromb Haemost. 2009;101:77-85.
Correspondência

Ricardo de Alvarenga Yoshida

Departamento de Cirurgia e Ortopedia da Faculdade de Medicina de Botucatu

da Unesp

Campus de Botucatu

CEP 18618-970 - Botucatu (SP), Brasil

E-mail: ricardoyoshida@gmail.com

Contribuições dos autores Concepção e desenho do estudo: RAY, WBY Análise e interpretação dos dados: RAY, WBY, HAR

Coleta de dados: RAY, WBY

Redação do artigo: RAY, WBY

Revisão crítica do texto: RAY, WBY, HAR

Aprovação final do artigo*: RAY, WBY, HAR, RED

Análise estatística: RAY, WBY

Responsabilidade geral pelo estudo: RAY

*Todos os autores leram e aprovaram a versão final submetida ao I Vasc Bras. 